\title{
Interdisciplinaridade e ensino superior na área da saúde: Perspectivas para a formação profissional
}

\author{
Interdisciplinarity and higher education in the health area: Perspectives for the professional \\ qualification
}

Interdisciplinaridad y educación superior en el área de salud: Perspectivas para el formación professional

\begin{abstract}
Resumo
Um dos maiores desafios atuais do Ensino Superior na área da Saúde refere-se ao compromisso com a formação de profissionais capacitados por meio de propostas inovadoras, sob o viés da articulação dos saberes e integração entre as disciplinas. Este trabalho consiste em uma revisão da literatura que aborda a temática "interdisciplinaridade na saúde", entendendo esta como uma nova atitude frente ao conhecimento, o que possibilita a transformação mútua, a ressignificação e a reestruturação das especialidades como fonte de autoconhecimento. Foram selecionadas 21 publicações que preencheram os critérios de inclusão. A análise dos diversos estudos permitiu concluir que a interdisciplinaridade proporciona compreensão da inter-relação entre as áreas profissionais com toda a sua pluralidade e complexidade. Apesar das fragilidades apontadas e dos desafios a serem vencidos devido à persistência do ensino tradicional fragmentado, as ações interdisciplinares podem ser praticadas desde que haja uma diretriz no projeto pedagógico comprometida com a inovação metodológica e com a construção de um novo saber e um novo fazer.
\end{abstract}

Palavras-chave: Interdisciplinaridade; Formação profissional; Saúde.

\begin{abstract}
One of the biggest current challenges of Higher Education in Health Area refers to the compromise with the formation of trained professionals with innovative proposals, under the bias of knowledges articulation and integration between disciplines. This work consists of a review of the literature that deals with the theme of "interdisciplinarity in health", understanding it as a new attitude towards knowledge, which enables mutual transformation, re-signification and restructuring of specialties as a source of self-knowledge. We selected 21 publications that met the inclusion criteria. The analysis of the several studies allows concluding that the interdisciplinarity provides an understanding of the interrelationship between the professional areas with all their plurality and complexity. In spite of the weaknesses pointed out and the challenges to be overcome due to the persistence of fragmented traditional teaching, interdisciplinary actions can be practiced provided there is a guideline in the pedagogical project committed to methodological innovation and the construction of a new knowledge and a new doing.
\end{abstract}

Keywords: Interdisciplinarity; Professional formation; Health.

\section{Resumen}

Uno de los mayores desafíos actuales de la Educación Superior en el área de la Salud se refiere al compromiso con la formación de profesionales capacitados a través de propuestas innovadoras, bajo el sesgo de la articulación del conocimiento y la integración entre disciplinas. Este trabajo consiste en una revisión de la literatura que aborda el tema “interdisciplinariedad en salud", entendiéndolo como una nueva actitud hacia el conocimiento, que posibilita la transformación mutua, el reencuadre y la reestructuración de las especialidades como fuente de autoconocimiento. Se seleccionaron 21 publicaciones que cumplieron con los criterios de inclusión. El análisis de los diferentes estudios permite concluir que la interdisciplinariedad permite comprender la interrelación entre áreas profesionales con toda su pluralidad y complejidad. A pesar de las debilidades señaladas y los desafíos a superar por la persistencia de la educación 
tradicional fragmentada, se pueden practicar acciones interdisciplinarias siempre que exista una pauta en el proyecto pedagógico comprometido con la innovación metodológica y la construcción de un nuevo conocimiento y un nuevo forma de hacer las cosas.

Palabras clave: Interdisciplinariedad; Formación professional; Salud.

\section{Introdução}

A orientação predominante na formação profissional é alheia à organização setorial e ao debate crítico sobre o cuidado na saúde, apresentando pouca ou quase nenhuma relação com a realidade social e epidemiológica da população. A presença de modelos curriculares fragmentados associada ao enfoque pedagógico restrito à transmissão de conhecimentos não privilegia a formação crítica do estudante.

Desde a consolidação do modelo tradicional (positivista) - composto por uma única disciplina - enquanto paradigma científico, os modelos de ensino e estudo orientam os profissionais a separarem e olharem as partes, tornando-os quase incapazes de perceber as relações entre elas. Com isso, as disciplinas se tornaram módulos de pesquisa e geraram respostas apenas para elas mesmas (Leite, Correia e Ruas, 2015).

A teoria cartesiana marca fronteiras entre as disciplinas e se dá desde o início da formação escolar até à formação universitária, dividindo-se em faculdades, e estas se separam em três grandes áreas: exatas, humanas e biológicas. Leite, Correia e Ruas (2015) afirmam que tais áreas não conseguem reconhecer os elos religantes existentes entre elas e dividem-se em disciplinas, que entram em uma disputa de poder.

De acordo com Fiorin et al., 2014, a formação superior dos profissionais de saúde foi historicamente construída, de modo geral, sobre a fragmentação de conteúdos e organizada em torno de especificidades. Na abordagem clássica de formação em saúde, o ensino é tecnicista e planejado segundo o referencial técnico-científico acumulado pelos docentes em suas respectivas áreas de especialidade ou dedicação profissional hegemônico e não em uma lógica de colaboração e complementaridade de saberes.

As teorias científicas mais tradicionais, baseadas no modelo cartesiano são limitadas e incapazes de fornecer soluções plausíveis para os problemas contemporâneos, cada vez mais complexos. “A crescente e rápida especialização tem conduzido à pulverização do conhecimento, à divisão dos saberes, tornando a formação dos profissionais de saúde deficitária e ineficaz para resolver os problemas de saúde da população" (Sousa, Bogo e Bastos, 2013).

A necessidade de recuperar a integridade do conhecimento leva a novas metodologias e a uma nova forma de explorar os saberes, em prol de uma formação profissional de melhor qualidade. Na busca por mudanças no sistema de ensino, exige-se uma reformulação para a adequação às necessidades atuais impostas pela sociedade, no sentido de atendimento das demandas sociais e na necessidade de repensar e adequar a formação profissional.

Desta forma, o presente artigo objetivou a discussão e análise das diferentes concepções de interdisciplinaridade - suas características e relações com o ensino acadêmico de nível superior da área da saúde - de forma a favorece o redimensionamento das relações entre os diferentes conteúdos, a superação da fragmentação dos conhecimentos e a formação do profissional com uma visão global do mundo.

\section{Metodologia}

Tratou-se de uma revisão bibliográfica narrativa, de caráter exploratório com abordagem qualitativa ampla e apropriada para discutir o referido tema sob uma perspectiva de condição fundamental do ensino e da pesquisa na sociedade contemporânea. Esta metodologia pode ser compreendida como um ponto de cruzamento entre atividade com lógicas diferentes e o seu enfoque reside na tentativa de extrapolar a mera justaposição das contribuições disciplinares, estabelecendo-se um intercâmbio entre especialistas de diversas áreas do conhecimento (Sousa, Bogo e Bastos, 2013; Velloso et al., 2016; Pereira, 2018). 
Foram relacionados artigos científicos indexados nas bases de dados: Scielo, Google Acadêmico e Latindex. O período de coleta de informações se deu durante o mês de Janeiro do ano de 2020. Para a sua realização, foram avaliados estudos realizados desde 1976, até os mais recentes realizados no ano de 2017.

Os critérios de inclusão foram artigos publicados no idioma Português, que se relacionaram ao tema "Ensino Interdisciplinar na formação dos profissionais da área da saúde", através do acesso on-line às referidas bases de dados. Realizouse a apresentação dos resultados de forma descritiva.

Assim, foram selecionadas 21 referências para a realização desse estudo. A estrutura do presente trabalho está organizada em uma revisão de literatura narrativa cujos critérios utilizados na avaliação e seleção dos trabalhos consultados não seguiram métodos sistemáticos de buscas (Bernardo; Nobre; Jatene, 2004).

\section{Desenvolvimento}

\subsection{Revisão de Literatura}

A formação superior na área da saúde está subsidiada por um discurso pedagógico que normatiza a relação de "poder" e "saber" diante do processo de saúde e doença. Essa relação é transmitida com base em um saber positivista, organicista e biologizante que valida e trata a saúde como ausência total de doença, de incapacidade e/ou de deficiência intrínseca e centrada no próprio indivíduo. A doença ainda é vista como uma condição que gera um desequilíbrio, uma desarmonia e uma falta de produtividade na vida diária humana.

Assim, o olhar clínico, ensinado e aprendido, diante da relação de saúde e doença, ainda objetiva ver no homem o produto do seu corpo, negando a possibilidade de fazer dele e de sua saúde um reduto de suas técnicas, modos de vida e de suas representações sociais (Leite, Correia e Ruas, 2015).

Fiorin et al. 2014 argumentam que o Ensino Superior no Brasil na área da Saúde vem sendo alvo de inúmeras críticas, questionando a formação dos profissionais acerca da qualidade da prática em saúde em um novo contexto social. A ruptura se originou no pensamento moderno, no início do século XVII. Com o advento da Idade Moderna, a ciência unitária rapidamente se rompeu e continuou nesse processo de dissociação ao longo de sua trajetória.

O desenvolvimento técnico-científico, decorrente da crescente e rápida especialização, tem levado à pulverização, ao esmigalhamento do conhecimento e à divisão dos saberes em diversas disciplinas, ou ramos do saber, originando novas especialidades e subespecialidades (Gattás, 2005). O método cartesiano impregnou fortemente o paradigma da ciência moderna, provocando a fragmentação do conhecimento, dividindo o saber em partes cada vez menores, representadas pelas disciplinas, contribuindo assim para a fragmentação do pensamento e da visão parcelar do mundo.

Sousa, Bogo e Bastos (2013) salientam que a formação superior dos profissionais de saúde ainda se baseia na forte tradição positivista e biocêntrica no tratamento dos problemas de saúde, nos espaços de poder que a disciplinarização significa, na rigidez das estruturas acadêmicas organizadas de forma vertical e em departamentos, no modelo técnico-linear ainda presente nos currículos, na falta de articulação entre teoria e prática, na centralidade no papel do professor nas salas de aula e na resistência de muitos docentes à mudança.

Como forma de compreender a complexidade dos fenômenos, de diminuir os efeitos nefastos da fragmentação do saber e de oferecer assistência humanizada pela percepção do homem em suas múltiplas dimensões, a interdisciplinaridade é apontada como fundamental tanto na área da educação, no preparo dos futuros profissionais da saúde, quanto nas atividades do cotidiano dos serviços de saúde (Azzi e Silva, 2000; Gattás, 2005; Souza, 2012).

"A interdisciplinaridade se caracteriza pela intensidade das trocas entre especialistas e pela integração das disciplinas num projeto comum, em que se estabelece uma relação de reciprocidade" (Velloso et al., 2016). Fiorin et al. (2014) reforçam ao 
afirmar que a interdisciplinaridade é concebida como uma integração de disciplinas, de áreas de conhecimento ou de profissionais, podendo facilitar o aprendizado, a organização do trabalho e a comunicação entre os diferentes cursos. A literatura, especialmente a sociológica, aponta a necessária atenção para os espaços institucionais, como mediadores na construção do conhecimento e de práticas inovadoras, destacando a interinstitucionalidade e interdisciplinaridade, via conexões e interdependência entre diferentes saberes e práticas. Ou seja, o campo da saúde envolve um diálogo constituído por um amplo leque de disciplinas, abrangendo as ciências da vida, passando pelas ciências físicas e do meio ambiente, chegando até as humanas e sociais, com a complexidade dos sujeitos e de suas relações sociais (Velloso et al., 2016).

Diante da importância em compreendermos a relação dinâmica entre o todo e suas partes, os saberes interdisciplinares - como os da saúde coletiva - deveriam ser direcionadores teóricos mais potentes em qualquer formação superior em saúde (Leite, Correia e Ruas, 2015).

Os referidos autores argumentam que a institucionalização dos saberes e sua organização em práticas se dariam mediante a conformação de núcleos e campos. Núcleos seriam aglutinação de conhecimentos e demarcariam a identidade de uma área de saber e de prática profissional; campos seriam um espaço de limites imprecisos onde cada disciplina e profissão buscariam em outros apoios para cumprir suas tarefas teóricas e práticas.

Então, compreendemos que, para a formação profissional na área de saúde, são necessárias ações interdisciplinares e multifacetadas entre as diversas disciplinas, que perpassam as especificidades desse campo com diversos núcleos profissionais, diálogos e ações constantes com os campos das ciências humanas e exatas. "Uma visão interdisciplinar, unificada e convergente implica estar presente tanto no campo da teoria como da prática, seja ela social, pedagógica ou de pesquisa" (Gattás, 2005). O termo "interdisciplinar" surgiu na área da Educação no final da década de 60, tendo como pano de fundo a insatisfação de professores e de alunos com os rumos que a área tomava na época. No topo de tais discussões (especialização acelerada, fragmentação do ensino, conhecimentos dissociados da prática) surgiram várias inovações nas universidades europeias, entre elas o recurso da interdisciplinaridade como elemento de superação da excessiva especialização.

Furlanetto (2011) ressalta que "uma pesquisa interdisciplinar, ao contrário da disciplinar que coloca os problemas os problemas em função das disciplinas, encontra seus critérios de pertinência no contexto preciso da situação em questão."

Com base nos posicionamentos dos estudiosos sobre o tema interdisciplinaridade (Reis, 2009; Rossit et al., 2012; Mainzer, 2011), conclui-se que disciplina é um conhecimento progressivo e especializado, que pode conduzir à visão aprofundada de um determinado campo de estudo ou de atuação. "Na mesma linha de reflexão, entende-se que a interdisciplinaridade é uma tentativa de superação dessa visão parcelar e dissociada da realidade" (Gattás, 2005).

Se o processo ensino-aprendizagem está organizado sob um molde dicotômico, a formação profissional também será fragmentada, o que dificulta o entendimento da necessidade de uma equipe integrada. Vivemos num mundo globalizado, o que torna os problemas e as soluções cada vez mais globais. Portanto, o momento atual exige ações em saúde e em educação interligadas às ciências humanas e exatas. Velloso et al. (2016) concluem que a complexidade do campo da Saúde Coletiva se deve ao fato de que os cuidados direcionados ao processo saúde-doença são complexos, possuem facetas e ângulos distintos e exigem múltiplos olhares para atingir os diferentes ângulos. Soma-se a isto o fato de que o nosso olhar recorta-os de uma maneira que é própria da nossa disciplina.

Conforme salienta Saupe et al. (2005), "a interdisciplinaridade constitui um entre os vários temas que necessitam ser desenvolvidos para gerar contribuição para a pauta da área da saúde”. Na perspectiva contemporânea, o tema da interdisciplinaridade contempla: o reconhecimento da complexidade crescente do objeto das ciências da saúde e a consequente exigência interna de um olhar plural; a possibilidade de trabalho conjunto, que respeita as bases disciplinares específicas, porém busca soluções compartilhadas para os problemas das pessoas e das instituições; o investimento como estratégia para a concretização da integralidade das ações de saúde (Saupe et al., 2005). 
Interdisciplinaridade tem sido objeto constante de discussão quando se aborda as ciências da saúde. Japiassu (1976) levantou a possibilidade de esse neologismo tomar sentidos amplos e diversificados, com consequentes entendimentos e usos.

"Um dos maiores desafios atuais do Ensino Superior refere-se ao compromisso com a formação de profissionais capacitados, que se caracteriza por meio de propostas inovadoras de formação" (Fiorin et al., 2014). Se esse educador entende a realidade complexa da organização social e do próprio sujeito, auxilia o aluno a perceber, criticamente, todo o seu entorno, subjetiva e objetivamente, ele irá ampliar a consciência do estudante sobre os elementos de sua própria historicidade. "Essa é uma das formas pelas quais se pode compreender melhor as percepções dos alunos, para a partir daí propor novas formas de enxergar, refletir e lidar com as demandas apresentadas" (Leite, Correia e Ruas, 2015). Os referidos autores salientam que a qualidade da formação superior não deve responder somente às dimensões do "aprender a fazer", mas também do "aprender a aprender" e do "aprender a ser" com uma implicação crítica, política, democrática e social para o melhor enfrentamento e compreensão teórico-prática das necessidades coletivas em saúde.

Conforme Reis (2009), um sistema educativo eficiente será aquele capaz de fornecer educação de qualidade numa perspectiva interdisciplinar, proporcionando a compreensão da inter-relação das diferentes áreas profissionais com toda a sua complexidade e pluralidade. Partindo dessa premissa, Fiorin et al. (2014) reforçam a ideia de que "a interdisciplinaridade vai além de uma justaposição ou adição de diferentes saberes. Ocorre, neste sentido, uma comunicação entre conteúdos e discussão sobre as perspectivas, estabelecendo interação entre si”.

O debate sobre a formação adequada dos profissionais de saúde e sobre a necessidade de modificações nas orientações curriculares enfatiza a elaboração, execução e avaliação participativa (gestores, professores e alunos) no desenvolvimento de métodos e estratégias pedagógicas inovadoras, valorizando o vínculo entre as universidades e os serviços de saúde, bem como a inclusão do princípio da integralidade/pluralidade como um eixo da formação em saúde (Soares e Aguiar, 2010).

A nova proposta de formação de profissionais de saúde representa uma alternativa avançada de estudos superiores que permitirão reunir um conjunto de características que hoje vêm sendo requeridas para a formação universitária profissional e cidadã na área da saúde (Mainzer, 2008).

Nessa perspectiva, propõem-se novas adequações com o intuito de integralizar saberes e construir conhecimentos através de ações interdisciplinares dentro das universidades, contextualizando o aluno em diversas situações do cotidiano que serão enfrentadas no âmbito profissional.

Conforme Leite, Correia e Ruas (2015), "a ação interdisciplinar nos leva a novas disciplinas, novas práticas, novos problemas". Dessa concepção, decorrem duas importantes consequências: o alargamento do conceito de ciência e a necessidade de reorganização das estruturas da aprendizagem das ciências, nomeadamente, a universidade. As ações interdisciplinares representam uma proposta de formação pautada na articulação de conceitos, percepções para a produção de saberes sobre práticas de ensino alicerçadas na integralidade e com a necessidade de se assumir uma postura crítica e criativa entre educação em saúde e trabalho em saúde (Leonello, Neto e Oliveira, 2011).

De acordo com os estudos de Mendes, Lewgoy e Silveira (2008), o ensino multiprofissional/interprofissional pode ser conceituado como uma proposta na qual várias profissões aprendem juntas sobre o trabalho conjunto e sobre as especificidades de cada uma, na melhoria da qualidade no cuidado ao paciente. A interdisciplinaridade deve ser pensada como base na formação inicial e continuada de profissionais na área da saúde.

Não se permite mais a fragmentação de conhecimentos, o ensino em blocos, teorias redutoras que fogem da lógica da compreensão do valor da cultura e do contexto social nas diversas vivências e experiências de agentes envolvidos nos processos de aprender e ensinar a realidade. 
Neste sentido, é válido destacar, conforme Silva (2011), que a inserção responsável e comprometida de estudantes e docentes nos cenários reais, desde a primeira série dos cursos visa à integração teórico/prática e ensino/serviço, podendo ser a base e a guia em torno do qual se tece uma nova forma de ser, fazer, conhecer e conviver em saúde.

O desafio da interdisciplinaridade é elaborar a ligação entre saberes, até agora desconectados, e às vezes antagônicos, mas que se religados ampliam a capacidade de percepção do sujeito, garantindo-lhe mais autonomia e mais capacidade de se entender numa rede complexa de diversos saberes. No momento atual, questiona-se: por que o enfoque interdisciplinar? Quais suas dificuldades e perspectivas? Como desenvolver profissionais de saúde voltados ao trabalho interdisciplinar/multiprofissional?

\section{Resultados e Discussão}

Diversos estudos sobre a relação entre interdisciplinaridade e formação profissional relatam que profissional relatam que a interdisciplinaridade não é uma mistura de disciplinas, mas um estudo profundo sobre determinado tema através de várias óticas, assumindo um papel de articulação dos saberes que se desdobram em diversos horizontes e várias possibilidades (Rossit et al., 2012), o que é consenso entre os diversos autores (Azzi e Silva, 2000; Galindo e Goldenberg, 2008; Japiassu, 1976; Souza et al., 2012).

Os mesmos questionamentos éticos, políticos e sociais que disparam o processo de transição entre os paradigmas científico-moderno e científico-social demandam um novo olhar e uma nova postura em relação à prática docente (Rossit et al., 2012). Nesse contexto, a interdisciplinaridade surge como uma nova ótica para a reformulação da produção, da organização e da disponibilidade do conhecimento (Horst e Orzechowski, 2017).

Em coerência com tais observações, Gattás (2005) argumenta que a interdisciplinaridade "é uma exigência da própria evolução do conhecimento e da criatividade de pesquisadores que buscam novos métodos de trabalho e sistemas de organização".

Em consonância com a tendência do novo paradigma de que as ciências devem interagir, a interdisciplinaridade entra com um instrumento facilitador, uma ferramenta indispensável no processo ensino-aprendizado (Rossit et al., 2012), o que está de acordo com os estudos de Batista (2008) e Furlanetto (2011).

Tais autores relacionam a docência universitária com a prática social, de forma a procurar a aprender novos saberes e novas práticas, em um contexto econômico, demográfico, temporal e espacial. Há um consenso entre os referidos achados e as pesquisas de Leite, Correia e Ruas (2015) e Horst e Orzechowski (2017) que afirmam que a área da saúde, por ser um núcleo complexo de vários saberes, exige um posicionamento ético-técnico-político frente à realidade e aos argumentos que a constroem, em oposição ao positivismo que reduz a apreensão das experiências entre saúde/doença e seus modos processuais de intervenção.

Furlanetto (2011) ressalta que a interdisciplinaridade apresenta inúmeras facetas que necessitam ser exploradas para que os sentidos atribuídos a ela possam ser compreendidos. O prefixo inter desloca a interdisciplinaridade do centro dos territórios disciplinares e a instala nas bordas. Ela está destinada a mover-se nas fronteiras de territórios separados, procurando descobrir permeabilidades no espaço do "entre" que permitam estabelecer novas relações. Tais estudos corroboram as pesquisas de Mendes, Lewgoy e Silveira (2008) que salientam que a interdisciplinaridade vai além de uma justaposição ou adição de diferentes ângulos sobre determinados objetos de análise. As disciplinas se comunicam umas com as outras, confrontam e discutem as suas perspectivas, estabelecendo entre si uma interação mais forte.

Ao considerar a concepção de saúde-doença na perspectiva da qualidade de vida, há a necessidade de se promover transformações na formação profissional, visando buscar a articulação entre teoria-prática e integração entre ensino-serviçocomunidade (Mainzer, 2011; Reis, 2009; Silva, 2008). 


\section{Conclusão}

O presente estudo aponta a crescente importância do enfoque interdisciplinar na formação profissional da área da saúde e revela a necessidade de se romper o ensino tradicional fragmentado e desarticulado com o atual contexto social. Ainda há um longo caminho a ser percorrido em busca de uma prática interdisciplinar que gere ações resolutivas na área da saúde.

O perfil das publicações analisadas remete ao discurso teórico sobre a interdisciplinaridade, essencial para instigar a reflexão, o senso crítico e criativo, pois permite aos envolvidos (professores, alunos, coordenadores, funcionários e usuários) uma percepção mais integralizada do conhecimento, da condição humana e da pluralidade dos fenômenos. Ela possui diversas facetas de ordem cognitiva, organizacional, administrativa e político-institucional. Sem a simultaneidade de tais elementos, o trabalho torna-se árduo, ineficaz e até contraditório.

A interdisciplinaridade é uma atividade que proporciona reflexões profundas e críticas, possibilitando a eliminação da distância entre formação escolar e atividade profissional.

Os movimentos simultâneos de buscar, registrar, refletir, avaliar e partilhar presidiram a elaboração deste trabalho. Sugere-se que vários outros estudos sejam realizados, replicando o modelo de vivência interdisciplinar aqui descrito.

\section{Referências}

Aguiar, K., Amorim, A. C., Batista, S. H., Costa, A., Maciel, A., Nobre, A. F., Regis, C., \& Rossit, R. A. S. (2012). Aprendizagem e Interdisciplinaridade na Saúde. ISBN $978-85-66540-03-1$. São Paulo.

Azzi, R. G., \& Silva, S. H. S. (2000). A importância de um "novo olhar" do professor para os alunos - um primeiro passo na busca de melhores resultados no processo ensino-aprendizagem. In: Sisto, F, Oliveira, G.G. e FINI, L.D.T; Leituras de Psicologia para Formação de Professores. Petrópolis: Vozes.

Batista, S. H. (2008). Interdisciplinariedad, Docencia Universitaria y Formación. Educación Médica Superior (Habana), $22: 7$.

Bernardo, W. M., Nobre, M. R. C. \& Jatene, F. B. (2004). A prática clínica baseada em evidências. Parte II: buscando as evidências em fontes de informação. Rev. Assoc. Med. Bras. 50(1).

Fiorin, P. B. G., Motta, G. A., Baldissera, F. G., Magalhães, C. R., Salamoni, B., \& Zaneti, I. C. (2014). O Ensino Interdisciplinar na Área da Saúde: Perspectivas para a formação e a atuação multiprofissional. Rev. Didática Sistêmica, 16(2):30-43.

Furlanetto, E. C. (2011). Interdisciplinaridade: um conhecimento construído nas fronteiras. International Studies on Law and Education, 8: 47-54.

Galindo, M. B., \& Goldenberg, P. (2008). Interdisciplinaridade na Graduação em Enfermagem: Um Processo em Construção. Rev. Bras. Enfermagem, 61 (1): 11-7.

Gattás, M. L. B. (2005). Interdisciplinaridade em Cursos de Graduação na Área da Saúde da Universidade de Uberaba - UNIUBE. Ribeirão Preto.

Horst, V. S. B., \& Orzechowski, S.T. (2017). O Desafio e potencialidade da interdisciplinaridade no atendimento à saúde. Laplage em Revista, 3(1):192-201. Japiassu, H. (1976). Interdisciplinaridade e patologia do saber. Rio de Janeiro: Imago.

Leite, F. C., Correia, R. L., \& Ruas, T. C. B. (2015). O desafio da interdisciplinaridade na Faculdade de Medicina do ABC. ABCS Health Sci, 40(3):337-342.

Leonello, V. M., Neto, M. V. M., \& Oliveira, M. A. C. (2011). A formação superior de Enfermagem no Brasil: uma visão histórica. Revescenferm USP, 45(2):1774-9.

Mainzer, K. (2011). Interdisciplinarity and innovation dynamics. On convergence of research, technology, economy, and society. 7(4): $275-289$.

Mendes, J. M. R., Lewgoy, A. M. B., \& Silveira, E. C. (2008). Saúde e interdisciplinaridade: mundo vasto mundo. Rev. Ciência Saúde, 1(1):24-32.

Pereira, A. S. et al. (2018). Metodologia da pesquisa científica [e-book]. Santa Maria. Ed. UAB/NTE/UFSM.

Reis, M. B. F. (2009). Interdisciplinaridade na prática pedagógica: um desafio possível. Revelli, 1(2):26-45.

Rossit, R. A. S., MacieL, A., Costa, A., Amorim, A. C., Nobre, A. F., Regis, C., Aguiar, K., \& Batista, S. H. (2012). Aprendizagem e interdisciplinaridade na saúde. Coletânea Mestrado Profissional Ensino em Ciências da Saúde - Região Norte / Universidade Federal de São Paulo. São Paulo.

Saupe, R., Cutolo, L. R. A., Wendhausen, A. L. P., \& Benito, G. A. V. (2005). Competência dos profissionais da saúde para o trabalho interdisciplinar. Interface - Comunic., Saúde, Educ., v.9, n.18, p.521-36.

Silva, R. H. A. (2011). Educação interprofissional na graduação em saúde: aspectos avaliativos da implantação na Faculdade de Medicina de Marília (Famema). Educrev, (39):159-75. 
Research, Society and Development, v. 10, n. 6, e22310615455, 2021

(CC BY 4.0) | ISSN 2525-3409 | DOI: http://dx.doi.org/10.33448/rsd-v10i6.15455

Soares, N. T., \& Aguiar, A.C. (2010). Diretrizes curriculares nacionais para os cursos de nutrição: avanços, lacunas, ambiguidades e perspectivas. Rev Nutr, 23(5): p. 895-905.

Sousa, I. F., Bogo, D., \& Bastos, P. R. H. O. (2013). Formação Interdisciplinar para Atuação no Sistema Único de Saúde: SÍNTESE DE ARTIGOS PUBLICADOS NO BRASIL. Rev. Saúde.com, 9(2):49-59.

Souza, M. C. A., Casotti, E., Mello, A. C. F., Goyatá, F. R., Souza, T.C., \& Albuquerque, C. J. M. (2012). Interdisciplinaridade no Ensino Superior: de Imagemobjetivo à Realidade! Revista Brasileira de Educação Médica, 36 (1, Supl. 2):158-163.

Velloso, M. P., Guimarães, M. B. L., Cruz, C. R. R., \& Neves, T. C. C. (2016). Interdisciplinaridade e formação na área de saúde coletiva. Trab. Educ. Saúde, 14(1):257-271. 